\title{
Transparent crystals with ultrahigh piezoelectricity
}

\section{Jurij Koruza}

\author{
It has been difficult to make transparent materials that have \\ extremely high piezoelectricity - a useful property related \\ to the coupling of electric fields and mechanical strain. This \\ hurdle has now been overcome. See p.350
}

\begin{abstract}
Piezoelectric materials show high electromechanical coupling, which means that they can generate large strains if an electric field is applied to them, and can transform external mechanical stimuli into electric charge or voltage ${ }^{1}$. They are widely used in electronic applications, including sensors, small motors and actuators - devices that convert electrical energy into movement. In addition, their high energy efficiency and ease of miniaturization are driving the development of new technologies, such as energy harvesters for the growing network of Internet-connected devices known as the Internet of Things, actuators for touch screens and microrobots. On page 350, Qiu et al. ${ }^{2}$ report the preparation of high-performance piezoelectrics that have the long-desired property of near-perfect transparency to light. This breakthrough could lead to devices that combine excellent piezoelectricity with tunable optical properties.

Most high-performance piezoelectrics
\end{abstract} are ferroelectrics - materials that have a spontaneous electric polarization that can be reversed by the application of an external electric field. At the atomic level, ferroelectrics have a local polarization that is caused by the displacements of certain ions from their symmetric positions. Regions that have a uniform direction of polarization are referred to as ferroelectric domains and are separated by boundaries called domain walls. The material's crystal structure determines the possible directions of polarization and, in turn, the types of domain wall. For example, a rhombohedral crystal structure enables 8 possible domain variants and $71^{\circ}, 109^{\circ}$ and $180^{\circ}$ domain walls (where the angle refers to the difference in polarization direction between the domains separated by the wall).

For these ferroelectrics to be used as piezoelectrics, they must first undergo a process known as poling, in which an external electric field is applied to the material to reorient unfavourably oriented domains and induce macroscopic polarization. Poled ferroelectrics show a large electromechanical response to an external electric field or to mechanical force, and this response is typically characterized by a quantity dubbed the piezoelectric coefficient. The magnitude of this coefficient depends to a large extent on the domain configuration; the other major contribution comes from the crystal lattice. Some of the largest coefficients known today were reported $^{3,4}$ for ferroelectric crystals based on lead magnesium niobate-lead titanate (PMN-PT). These crystals have piezoelectric coefficients above 1,500 picocoulombs per newton $\left(\mathrm{pC} \mathrm{N}^{-1}\right.$ ), which is about ten times higher than those of most other ferroelectrics.

The poling process is conventionally carried

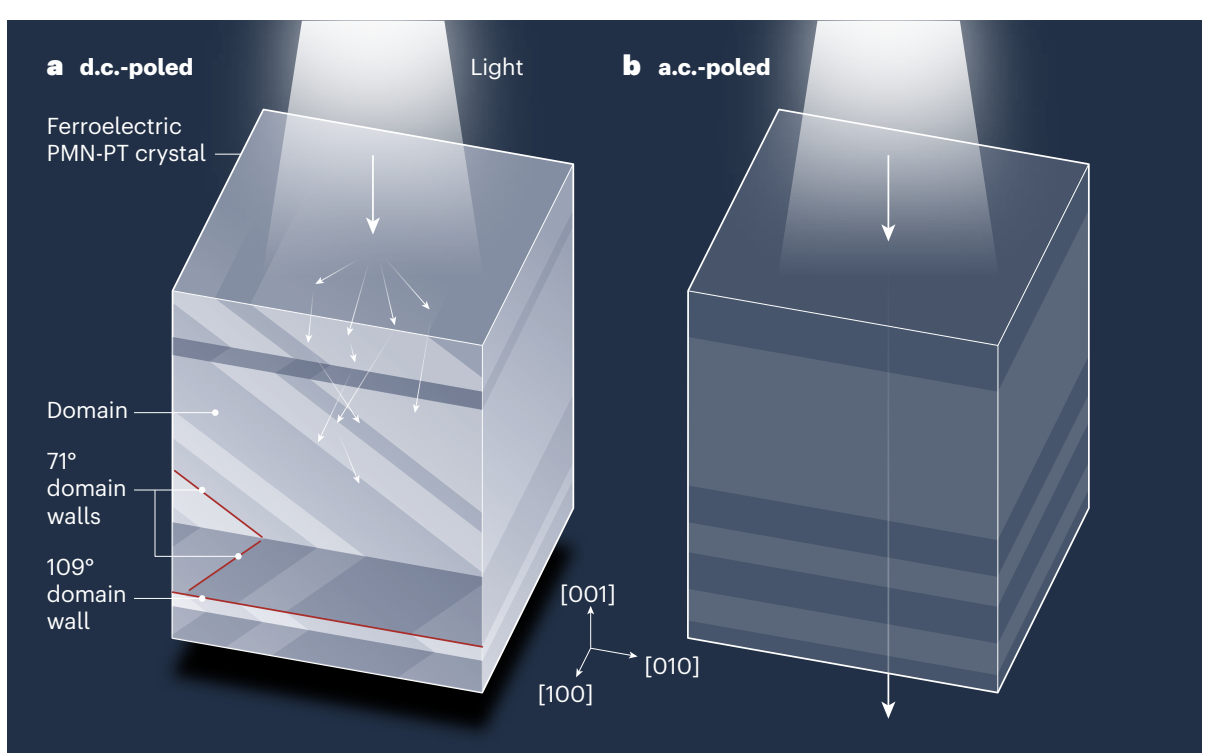

Figure 1 | Near-perfect light transmittance in a high-performance piezoelectric. a, Materials known as ferroelectrics contain regions of uniform electric polarization called domains (the different shades represent different orientations of polarization). These domains are separated by boundaries dubbed domain walls. When [001]-oriented rhombohedral ferroelectric crystals of lead magnesium niobate-lead titanate (PMN-PT) undergo a process called poling using direct-current (d.c.) electric fields, they contain both $71^{\circ}$ and $109^{\circ}$ domain walls (where the angle indicates the difference in polarization orientation between the domains separated by the wall). The $71^{\circ}$ walls cause incident light to be scattered, such that the crystals are opaque. b, Qiu et al. ${ }^{2}$ demonstrate that, when the poling is carried out using alternating-current (a.c.) electric fields, the number of $71^{\circ}$ domain walls is greatly reduced. The crystals show near-perfect light transmittance and ultrahigh piezoelectricity - a property associated with the coupling of electric fields and mechanical strain. 


\section{News \& views}

grown by a modified version of a widely used approach called the Bridgman method. They studied the domain structure of the crystals using three techniques (high-resolution X-ray diffraction, polarized-light microscopy and birefringence imaging spectroscopy), and confirmed the removal of $71^{\circ}$ domain walls throughout the a.c.-poled samples. These samples exhibited near-perfect light transmittance, large birefringence (an effect in which a material's refractive indices are different along different axes) and ultrahigh piezoelectric coefficients, exceeding 2,100 $\mathrm{pC} \mathrm{N}^{-1}$. For comparison, ferroelectrics that have a similar level of transparency to these crystals, such as lithium niobate or polyvinylidine fluoride, typically have piezoelectric coefficients ${ }^{1}$ of less than $40 \mathrm{pC} \mathrm{N}^{-1}$.

The report by Qiu et al. adds the optical component to high-performance piezoelectric crystals and therefore opens the door to the design of electro-optical-mechanical devices. Transparent actuators or motors could be used for touch screens in consumer electronics or for the development of invisible microrobots. Moreover, the crystals' high

\section{"Transparent actuators or motors could be used for touch screens or for the development of invisible microrobots."}

electromechanical performance and transparency could be harnessed in an imaging technology known as photoacoustic imaging or in piezoelectric light guides.

Although the authors demonstrated the applicability of their approach to several PMN-based crystals, it remains to be seen whether similar principles can be applied to other ferroelectric systems. Of particular interest are systems that remain polarized at high temperatures (above $100-150^{\circ} \mathrm{C}$ ), unlike PMN-based materials. Nevertheless, Qiu and colleagues' discovery has arrived just in time to meet the growing demand for multitasking smart materials and hybrid devices.

Jurij Koruza is at the Institute of Materials Science, Technische Universität Darmstadt, 64287 Darmstadt, Germany.

e-mail: koruza@ceramics.tu-darmstadt.de

1. Heywang, W., Lubitz, K. \& Wersing, W. (eds) Piezoelectricity: Evolution and Future of a Technology (Springer, 2008).

2. Qiu, C. et al. Nature 577, 350-354 (2020).

3. Park, S.-E. \& Shrout, T. R. J. Appl. Phys. 82, 1804-1811 (1997).

4. Zhang, S. et al. Prog. Mater. Sci. 68, 1-66 (2015).

5. Yamamoto, N., Yamashita, Y., Hosono, Y., Itsumi, K. \& Higuchi, K. US Patent 2014/0062261 A1 (2014).

6. Yamashita, Y., Yamamoto, N., Hosono, Y. \& Itsumi K. US Patent 2015/0372219 A1 (2015).

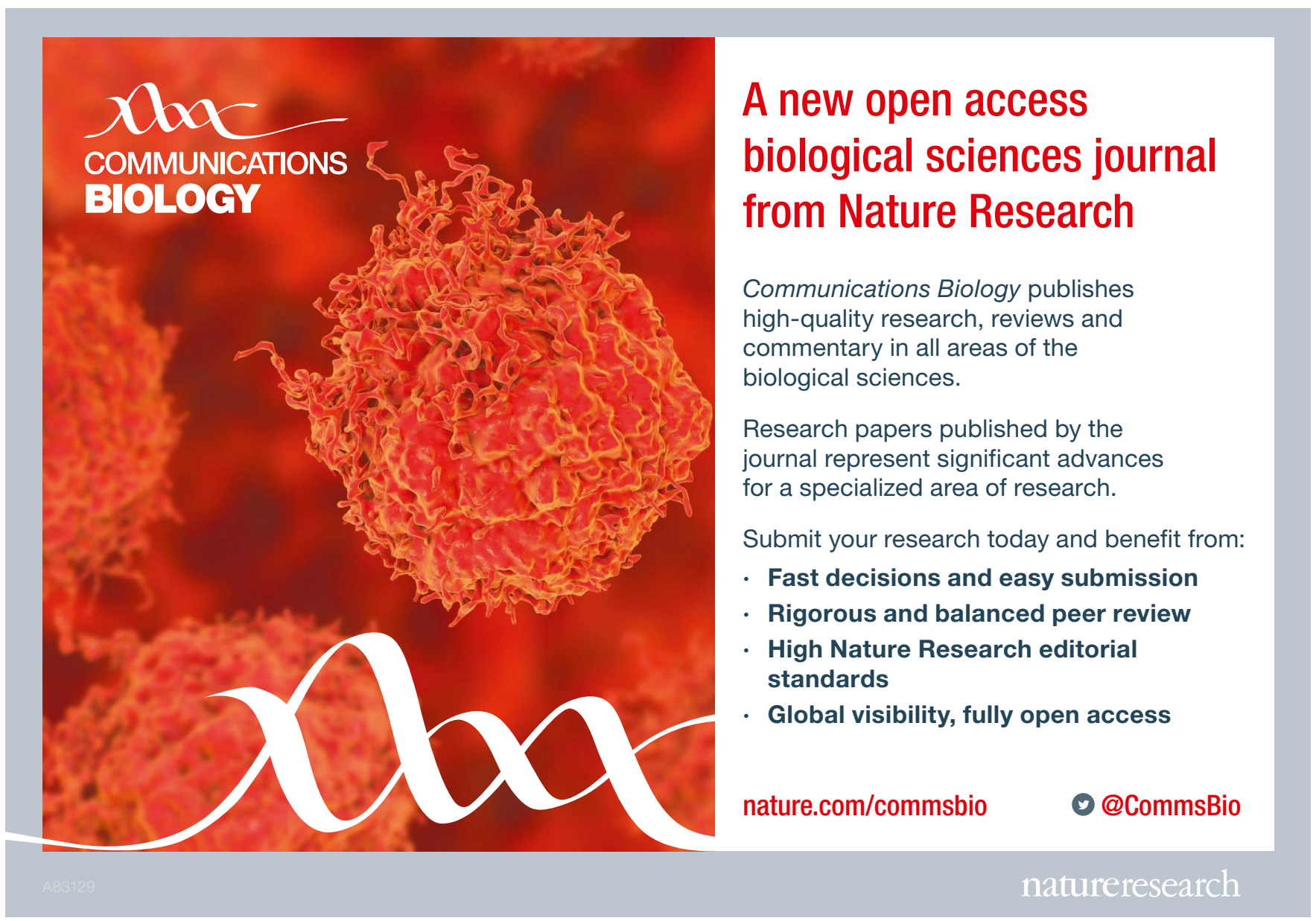

